\title{
LETTER
}

\section{Simplified immune-dysregulation index: a novel marker predicts 28-day mortality of intensive care patients with COVID-19}

\author{
Hui Chen ${ }^{1}$, Jun Wang ${ }^{1}$, Nan Su${ }^{1}$, Xiebing Bao ${ }^{2,3}$, Yongsheng $\mathrm{Li}^{4^{*}}$ and Jun Jin ${ }^{1 *}$ (D)
}

(C) 2020 Springer-Verlag GmbH Germany, part of Springer Nature

Dear Editor,

Since December 2019, a coronavirus disease-2019 (COVID-19) was first reported in Wuhan, China, which was caused by severe acute respiratory syndrome coronavirus 2 (SARS-CoV-2) and expeditiously spread to countries worldwide with massive mortality [1]. The response of lymphocytes in COVID-19 is characterized by a phenomenon of lymphopenia and hypercytokinemia, and is correlated with disease severity and prognosis [2]. Besides, interleukin-6 (IL-6) may be the key cytokine leading to inflammatory storm in COVID-19 [3]. Hence, we develop a simplified immune-dysregulation indexthe level of IL-6 to lymphocytes count ratio-to define the immune-dysregulation in COVID-19. We aimed to evaluate the predictive value of IL-6/lymphocyte in patients with COVID-19.

We conducted a retrospective study based on patients with confirmed diagnosis of COVID-19 admitted to the intensive care units in Tongji Hospital. The inclusion and exclusion criteria were presented in the online supplement. IL-6 $(\mathrm{pg} / \mathrm{ml})$ and lymphocytes count $\left(10^{6} / \mathrm{ml}\right)$ within the first day after hospital admission were collected. IL-6/lymphocyte was calculated. We used the highest ratio if a variable was recorded more than once. The primary outcome was 28-day mortality. Multivariate regression was selected to characterize the association

\footnotetext{
*Correspondence: dr_ysli@126.com; jinjun0514@163.com

1 Department of Intensive Care Medicine, The First Affiliated Hospital of Soochow University, No. 899 Pinghai Road, Suzhou 215000, Jiangsu, China

${ }^{4}$ Department of Intensive Care Medicine, Tongji Hospital, Tongji Medical College, Huazhong University of Science and Technology, 1095 Jiefang Avenue, Wuhan 430030, Hubei, China

Full author information is available at the end of the article
}

between IL-6, lymphocyte, IL-6/lymphocyte and 28-day mortality, respectively (description in the online supplement), we then model the nonlinear relationship between IL-6/lymphocyte and 28-day mortality using splines, and the predictive value of IL-6/lymphocyte, IL-6 and lymphocytes for 28-day mortality was compared according to receiver operating characteristic (ROC) curves, net reclassification improvement (NRI) and integrated discrimination improvement (IDI). We also attempt to stratified patients through the IL-6/lymphocyte. The study was approved by Tongji Hospital Ethics Committee.

A total of 172 patients were included in our study (description in the online supplement). Overall 28- day mortality was $50.5 \%$. Baseline characteristics between survivors and non-survivors are presented in eTable 1. Survivors had a higher lymphocytes count $(0.94$ ( $0.73-$ $1.28)$ vs. $0.53(0.38-0.74) ; p<0.001)$ and a lower IL-6 (7.1 (2-21.6) vs. $61.1(33.4-137.4) ; p<0.001)$ compared nonsurvivors. Statistical analysis demonstrated that lymphocyte is negatively correlated with IL-6 $(R=-0.26$; $p<0.001$ ) (eFig. 1). IL-6/lymphocyte was significantly higher in non-survivors than that in survivors (125.5 $(56.9-267.4)$ vs. $8.3(2.1-23.4) ; p<0.001)$. The logistic regression analyses showed a significantly association between lymphocyte (OR 0.03; 95\% CI 0.01-0.17; $p<0.001$ ), IL-6 (OR 1.03; 95\% CI 1.01-1.05; $p<0.001$ ); IL-6/lymphocyte (OR 1.05; 95\% CI 1.03-1.08; $p<0.001$ ) and 28-day mortality, respectively (eTables 2,3 and 4). The nonlinear relationship between IL-6/lymphocyte and 28-day mortality is shown in Fig. 1a.

Figure $1 \mathrm{~b}$ indicates that IL-6/lymphocyte had a higher area under the curve (AUC) $(0.93$ (95\% CI 0.9-0.96)) than IL-6 (0.88 (95\% CI 0.83-0.92); $p<0.001)$ and lymphocytes (0.81 (95\% CI 0.75-0.87); $p<0.001)$. Compared

\section{Springer}



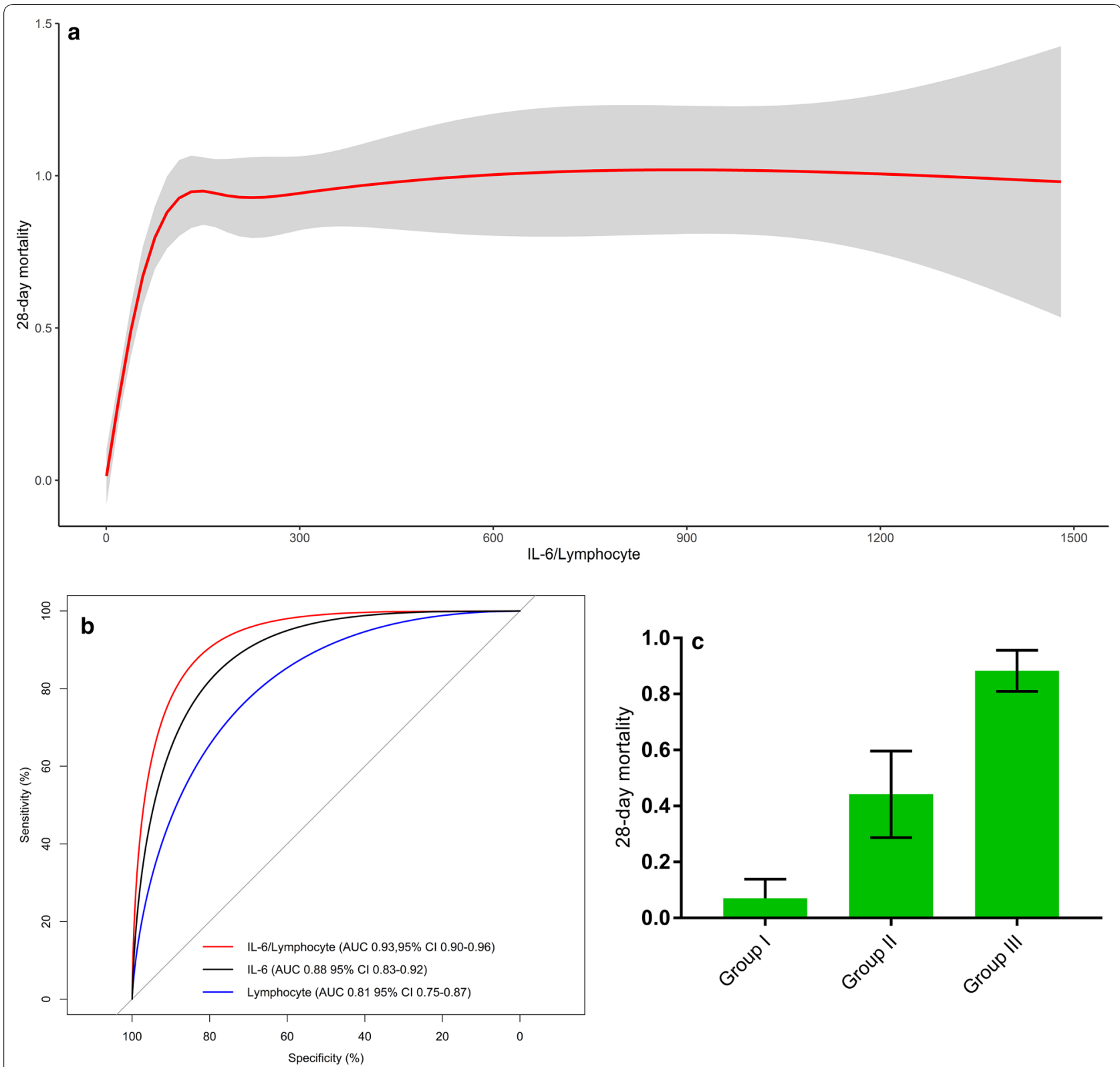

Fig. 1 a Relationship between IL-6/lymphocyte and 28-day mortality for intensive care patients with COVID-19 using splines. Overlying red is fitted linear splines with $95 \% \mathrm{Cl}$. b ROC curve analysis and comparison of the AUCs for IL-6/lymphocyte, IL-6 and lymphocytes in COVID-19. c Stratified mortality of groups based on the cutoff value of IL-6/lymphocyte in COVID-19. Bars show the percent of patients who died and 95\% CI. COVID-19: coronavirus disease-2019; ROC: receiver operating characteristic; AUC: area under the curve

with IL-6, IL-6/lymphocyte resulted in an additive NRI of $22.2 \%$ (95\% CI: 9.7-34.7\%; $p<0.001$ ), and an IDI of $11.8 \%$ (95\% CI: $8-15.6 \%$; $p<0.001$ ); compared with lymphocyte, IL-6/lymphocyte resulted in an additive NRI of $36.5 \%$ (95\% CI $17.2-55.8 \% ; p<0.001$ ) and an IDI of $20.2 \%$ (95\% CI 11.8-28.6\%; $p<0.001)$. According to the value of IL-6/lymphocyte, we divided patient into three groups: Group I (<15); Group II (15-50); Group III ( $>50)$, and the Group III had the highest 28-day mortality (Fig. 1c).
The degree of lymphopenia and the increase in inflammatory cytokine-especially IL-6-were associated with poor outcome in patients with COVID-19 [4]. Our study developed a novel marker to represent immunedysregulation and concluded that IL-6/lymphocyte had an improved predictive value compared to IL-6 and lymphocytes alone. Additionally, IL-6/lymphocyte showed a potential value to identify high-risk patients who needed more timely therapy at the early phase of COVID-19. 
The limitations of study are the incomplete assessment of immune-dysregulation and a single-center experience. Further researches are needed to compare IL-6/lymphocyte and other immunological indicators, as well as the dynamic change of IL-6/lymphocyte.

\section{Electronic supplementary materia}

The online version of this article (https://doi.org/10.1007/s00134-020-06114-2) contains supplementary material, which is available to authorized users.

\section{Author details}

${ }^{1}$ Department of Intensive Care Medicine, The First Affiliated Hospital of Soochow University, No. 899 Pinghai Road, Suzhou 215000, Jiangsu, China. ${ }^{2}$ National Clinical Research Center for Hematologic Diseases, Jiangsu Institute of Hematology, The First Affiliated Hospital of Soochow University, Suzhou, China. ${ }^{3}$ Institute of Blood and Marrow Transplantation, Collaborative Innovation Center of Hematology, Soochow University, Suzhou, China. ${ }^{4}$ Department of Intensive Care Medicine, Tongji Hospital, Tongji Medical College, Huazhong University of Science and Technology, 1095 Jiefang Avenue, Wuhan 430030, Hubei, China.

\section{Acknowledgements}

We thank all patients included in this study.

\section{Author contributions}

$J J$ and YSL conceptualized the research aims, design the study, and take responsibility for the integrity of the data and the accuracy of the data analysis. HC, NS and JW contributed to the acquisition of data, $\mathrm{HC}$ and XBB performed the statistical analysis. $\mathrm{HC}$ wrote the first draft of the paper, and other authors provided comments and approved the final manuscript.

\section{Funding}

This work was supported by Fundamental Research Funds for the central Universities (HUST: 2017KFYXJJ113) and Wuhan Municipal Science and Technology Bureau (2017060201010173) and The Emergency Project for the Prevention and Control of the Novel Coronavirus Outbreak in Suzhou, Jiangsu
Province, China, and Science Foundation of Jiangsu Commission of Health (H2018117)

\section{Compliance with ethical standards}

\section{Conflicts of interest}

The authors declare that they have no competing interests.

\section{Ethic approval and consent to participate}

The study was approved by Tongji Hospital Ethics Committee.

\section{Consent for publication}

Not applicable.

\section{Publisher's Note}

Springer Nature remains neutral with regard to jurisdictional claims in published maps and institutional affiliations.

Accepted: 12 May 2020

Published online: 20 May 2020

\section{References}

1. Wang D, Hu B, Hu C et al (2020) Clinical characteristics of 138 hospitalized patients with 2019 novel coronavirus-infected pneumonia in Wuhan, China. JAMA. https://doi.org/10.1001/jama.2020.1585

2. Xu Z, Shi L, Wang Y et al (2020) Pathological findings of COVID-19 associated with acute respiratory distress syndrome. Lancet Respir Med. https:// doi.org/10.1016/s2213-2600(20)30076-X

3. Xu X, Han M, Li T et al (2020) Effective treatment of severe COVID19 patients with tocilizumab. Proc Natl Acad Sci USA. https://doi. org/10.1073/pnas.2005615117

4. Ruan Q, Yang K, Wang W, Jiang L, Song J (2020) Clinical predictors of mortality due to COVID-19 based on an analysis of data of 150 patients from Wuhan, China. Intensive Care Med. https://doi.org/10.1007/s0013 4-020-05991-x 